OPEN ACCESS

Edited by:

José Mordoh,

Fundación Instituto Leloir,

Argentina

Reviewed by:

Daniel Olive,

Institut national de la santé et de la recherche médicale, France Florencia Paula Madorsky Rowdo,

Fundación Instituto Leloir,

Argentina

${ }^{*}$ Correspondence:

Arvind Chhabra

chhabra@uchc.edu

Specialty section: This article was submitted to Vaccines and Molecular

Therapeutics,

a section of the journal

Frontiers in Immunology

Received: 16 June 2017 Accepted: 03 August 2017 Published: 18 August 2017

Citation:

Chhabra A (2017) Inherent Immunogenicity or Lack Thereof of Pluripotent Stem Cells: Implications for Cell Replacement Therapy.

Front. Immunol. 8:993. doi: 10.3389/fimmu.2017.00993

\section{Inherent Immunogenicity or Lack Thereof of Pluripotent Stem Cells: Implications for Cell Replacement Therapy}

\author{
Arvind Chhabra* \\ Department of Medicine, University of Connecticut Health Center (UCONN Health), Farmington, CT, United States
}

Donor-specific induced pluripotent stem cells (iPSCs) offer opportunities for personalized cell replacement therapeutic approaches due to their unlimited self-renewal potential and ability to differentiate into different somatic cells. A significant progress has been made toward generating IPSC lines that are free of integrating viral vectors, development of xeno-free culture conditions, and differentiation of pluripotent stem cells (PSCs) into functional somatic cell lineages. Since donor-specific iPSC lines are genetically identical to the individual, they are expected to be immunologically matched and these iPSC lines and their cellular derivatives are not expected to be immunologically rejected. However, studies in mouse models, utilizing rejection of teratomas as a model, have claimed that syngenic iPSC lines, especially the iPSC lines derived with integrating viral vectors, could be inherently immunogenic. This manuscript reviews current understanding of inherent immunogenicity of PSC lines, especially that of the human IPSC lines and their cellular derivatives, and strategies to overcome it.

Keywords: human pluripotent stem cells, induced pluripotent stem cells, dendritic cells, immunogenicity, cell replacement therapy

\section{MAJOR ADVANCES IN PLURIPOTENT STEM CELL (PSC) RESEARCH FIELD}

Isolation of human embryonic stem cells (hESCs) from early stage embryos (1) and reprogramming of adult somatic cells into induced pluripotent stem cell (iPSC) lines (2-4) have opened up unique opportunities for developing patient-specific cell replacement therapies (CRT), novel drug discovery platforms, and understanding the mechanism of human cell lineages and organ development. Several different types of somatic cells, including terminally differentiated human primary immune cells, have now been successfully reprogrammed and many non-integrating virus-based iPSC derivation approaches have been developed. Among these include methods utilizing recombinant plasmids, episomes, non-integrating viruses such as adenoviruses and sendai viruses, mRNA, and protein delivery-based reprogramming methods that do not require any carrier vector to deliver the reprogramming factors and the microRNA-based reprogramming that does not require exogenous delivery of the reprogramming factors. Several different somatic cell lineages have been derived from donor-specific iPSC lines and a significant progress has been made toward their phenotypic and functional characterization. Significant progress has also been made toward characterizing the

Abbreviations: hPSCs, human pluripotent stem cells; hESCs, human embryonic stem cells; EB, embryoid bodies. 
mechanism of development and maintenance of pluripotency, effect of reprogramming on genetic and epigenetic landscape of somatic cells, and characterization of the inherent immunogenicity of iPSC lines and their cellular derivatives. However, much remains to be learnt as these issues are critical for generating iPSC lines that have stable genetic and epigenetic profiles and do not exhibit inherently immunogenicity. We here review current understanding of the inherent immunogenicity of PSC lines and their cellular derivatives, potential mechanisms behind inherent immunogenicity of PSC lines, and strategies to overcome potential immune rejection of PSC lines and their cellular derivatives, with a special emphasis on human hESC and iPSC lines and their cellular derivatives.

\section{INHERENT IMMUNOGENICITY OF MOUSE PSC BASED ON TERATOMA REJECTION}

Since iPSC lines are the genetic replica of somatic cells they are derived from, donor-specific iPSC lines are expected to be immunologically matched to the donor and the cellular derivatives derived from them are therefore not expected be immune rejected, unlike allogenic tissue and organ transplants. However, failure of syngenic mouse iPSC lines to generate teratomas, a method used to validate the differentiation potential of PSC lines, in immune competent mice was recently used as a readout for their inherent immunogenicity by Zhao et al. (5). Authors first showed that B6 syngenic mESC could form teratomas in immune competent B6 mice without any detectable T cell infiltration, but allogenic 129/sv mESC could not, unlike the SCID mice where both the mESC could form teratomas. Subsequently, authors used syngenic iPSC lines to generate teratomas and found that the iPSC lines derived by genome integrating retrovirus, the V-iPSC $(17 \%)$, were significantly less efficient in generating teratomas than the non-integrating episomal vector-derived E-iPSC (80\%) and the teratomas that did form were infiltrated with $\mathrm{T}$ cells, thereby concluding that while both the syngenic iPSC lines were immunogenic, $\mathrm{V}$-iPSCs were more immunogenic than the E-iPSCs (5). By comparing the gene expression profiles of ESCand E-iPSC-induced teratomas, authors identified nine genes that were over-expressed in iPSC-induced teratomas and found teratoma antigen-specific functional T cells in these animals, suggesting that rejection of iPSC-induced teratomas was mediated by teratoma-associated antigen-directed $\mathrm{T}$ cell responses, as the $\mathrm{CD} 4^{-/-}$and $\mathrm{CD}^{-1-}$ mice could form teratomas (5).

These findings raised significant concerns about the safety of iPSC (6); however, Araki et al. subsequently reported negligible inherent immunogenicity in mESC, syngenic mouse iPSC lines, and their cellular derivatives (7). Authors used seven iPSC lines and five mESC lines and found no statistical differences in their ability to form teratomas or $\mathrm{T}$ cell infiltration in teratomas formed from these iPSC lines in 10 independent experiments (7). Authors used chimeric mice derived from the iPSC and mESC lines and engrafted dermal and bone marrow tissues to examine host immune reaction against the iPSC-derived cellular derivatives in syngenic C57Bl6 and allogenic Balb-c mice and showed that the syngenic skin grafts could sustain for more than 10 weeks without much $\mathrm{T}$ cell infiltration but the allogenic grafts could not. Engraftment of GFP+ve iPSC or mESC-derived mice bone marrow in wild-type C57Bl6 animals, with or without prior irradiation, also achieved long-term engraftment of engrafted bone marrow-derived T cells, B cells, and granulocytes alongside host cells without any signs of phenotypic abnormalities or immune reactivity (7). Although authors did find $\mathrm{T}$ cell infiltration in engrafted cardiomyocytes and the size of teratomas in wild-type BL-6 animals was slightly smaller than in SCID mice, authors did not find any significant differences in inherent immunogenicity profiles of mouse ESC and iPSC lines (7). In agreement with Araki et al. (7), Guha et al. also reported lack of immunogenicity in syngenic C57BL6 mouse iPSC lines by showing that the subcapsular renal space engrafted syngenic iPSC could form teratomas, but allogenic iPSC could not (8). Using in vitro proliferation as a readout for $\mathrm{T}$ cell response, authors also did not find any difference in the $\mathrm{T}$ cell activation profiles of the animals before or after iPSC and ESC engraftment (8).

Interestingly, Todorova et al. have attributed lack of immune rejection observed in renal space system by Guha et al. (8) to the immature phenotype of APC present in the renal space, as coadministration of APC could result in teratoma rejection (9). Furthermore, utilizing humanized mice Zhao et al. supported their initial findings by demonstrating that human iPSC-derived cellular derivatives exhibit differences in their immunogenicity profiles that correlate with their immunogenic antigen profiles (10). Supporting Zhao et al., de Almeida et al. have also reported rejection of mouse iPSC lines (11). Table 1 lists studies reporting immunogenicity or lack thereof of in ESC and iPSC lines. These findings have highlighted the need to characterize the inherent immunogenicity profile of human iPSC lines and their cellular derivatives to develop safe and effective CRT.

\section{IS CELL NUMBER A CRITICAL FACTOR IN FACILITATING DEVELOPMENT OF PSC-INDUCED TERATOMA?}

Interestingly, there are significant differences in the number of transplanted cells used for teratoma induction in abovementioned studies claiming inherent immunogenicity of mouse iPSC lines or lack thereof. While Zhao et al. in their first study used "one or three million" PSC for teratoma induction, without specifying which lines were used at 1 million and which ones at 3 million (5), in their subsequent study in humanized mice

\begin{tabular}{lllr}
\hline TABLE $\mathbf{1}$ & Studies reporting immunogenicity or lack of it in ESC and iPSC lines. \\
\hline S. No. & Study & $\begin{array}{l}\text { Immunogenicity of } \\
\text { syngenic mouse-ESC } \\
\text { and iPSC lines }\end{array}$ & $\begin{array}{r}\text { Cells used } \\
\text { for teratoma } \\
\text { induction }\end{array}$ \\
\hline 1. & Zhao et al. (5) & Immunogenic & 1 or $3 \times 10^{6}$ \\
2. & Araki et al. (7) & Non-immunogenic & $3 \times 10^{6}$ \\
3. & Guha et al. (8) & Non-immunogenic & $1 \times 10^{6}$ \\
4. & Zhao et al. (10) & Immunogenic & $2-4 \times 10^{6}$ \\
5. & de Almeida et al. (11) & Immunogenic & $1 \times 10^{6}$
\end{tabular}


authors used $2-4 \times 10^{6}$ cells (10). Araki et al. used $3 \times 10^{6}$ cells (7) and Guha et al. used $1 \times 10^{6}$ in their renal space teratoma induction experiments (8). In this context, it should be pointed out that Koch et al. have shown that the number of PSC used for teratoma induction is an important factor in determining generation of autologous versus allogenic teratomas, such that $1 \times 10^{6}$ allogenic mESC always fail to induce teratoma, $5 \times 10^{6}$ allogenic mESC can form teratomas in some animals (30\%), but $20 \times 10^{6}$ allogenic mESC can form teratomas in all the transplanted animals (12). In their humanized mice study, Zhao et al. also reported that only one in six animals injected with $2 \times 10^{6}$ hESC could form teratomas, while all the animals injected with $4 \times 10^{6}$ hESC were able to form teratomas (10). Using $1 \times 10^{6}$ mouse iPSCs, de Almeida also reported failure of these cells to form teratoma (11). Therefore, it is possible that the variability in teratoma formation in these studies is in part due to differences in number of PSC transplanted and an optimum PSC dosage for teratoma induction experiments must be identified to avoid experimental variations.

\section{IMMUNOSUPPRESSIVE PROPERTIES OF MOUSE ESC AND IPSC LINES}

Mouse ESC have been shown to exhibit immunosuppressive properties (12) that has also been confirmed in hESC and human iPSC lines $(13,14)$. The mESC express mRNAs for MHC molecules but not the corresponding proteins (15), and they can inhibit $\mathrm{T}$ cell proliferation and prevent LPS-mediated induction of co-stimulatory molecules in dendritic cells (DCs) in part through a TGF- $\beta$-mediated process (12). However, T cells cultured in the presence of $\mathrm{mESC}$ can be stimulated following purification suggesting that the mESC-mediated immune suppression is reversible and it does not make T cells anergic. Natural killer (NK) cells target MHC negative cells, but the published data on NK cell-mediated killing of mESC are not clear, as Koch et al. showed that mESC are not susceptible to NK cell-mediated killing (12) while Dressel et al. found that IL-2 activated syngenic, allogenic, and xenogenic NK cells could efficiently kill mESC (16). As mentioned before, systematic characterization of the inherent immunogenicity profile of human iPSC lines, especially the iPSC lines derived from different somatic cell sources and with different iPSC derivation methods, is essential for developing safe and effective CRT, since mouse iPSC lines derived from different somatic cell sources have been shown to harbor somatic cell memory and exhibit differences in their differentiation profiles $(17,18)$.

This is also important in light of the fact that despite the reprogramming factors discovered in mouse (2) are also sufficient to reprogram human somatic cells (3), significant differences have been found in the downstream genes targeted by these reprogramming factors in mice and humans (19). Ginis et al. reported species-specific differences in cell cycle regulation, apoptosis regulation, and cytokine expression profiles of human and mouse ESC lines (20), and Suh et al. identified 36 microRNAs in hESC that were downregulated in embryoid bodies (EB), some of which were shared with mouse ESC while others were specific for the hESC (21). Differences in mouse and human physiology are also well known now (22). For example, human blood is neutrophil rich (50-70\% neutrophils, 30-50\% lymphocytes) while mouse blood is lymphocyte rich (75-90\% lymphocytes, 10-25\% neutrophils) (22). Mouse HSC are c-kit ${ }^{\text {high }}$, flt- $3^{-}$while human HSC are c-kit ${ }^{\text {low }}$, flt- $3^{+}(23)$. Humans express 10 toll-like receptors (TLRs) and 22 NOD-like receptors (NLRs), while mice express 12 TLRs and 34 NLRs. Thy1 is a marker for mouse but not human T cells (24), interleukin-7 receptor (IL-7R) deficiency results in loss of both $\mathrm{T}$ and $\mathrm{B}$ cells in mouse (25) but only of T cells in humans (26) and Zap-70 deficiency results in loss of both CD4 ${ }^{+}$ and $\mathrm{CD}^{+} \mathrm{T}$ cells in mouse but only of $\mathrm{CD}^{+} \mathrm{T}$ cells in humans (27). Caspases also exhibit notable differences, as mouse do not express caspase-10 while humans do and deletion of caspase- 8 causes embryonic lethality in mouse but immune deficiency in humans $(28,29)$. Furthermore, promising treatment modalities in preclinical models do not always produce matching outcomes in humans $(30,31)$. These differences emphasize that the species variability must be taken into consideration before extrapolating findings in animal models to humans. We now review current understanding of the inherent immunogenicity of hESC and human iPSC lines.

\section{IMMUNOGENICITY OF HUMAN hESC AND IPSC LINES}

The issue of inherent immunogenicity of human pluripotent stem cell (hPSC) lines was first examined with hESC lines, and they were shown to express low levels of MHC class I molecules but no MHC class II molecules (32). Drukker et al. transplanted $1 \times 10^{6} \mathrm{hESC}$ in different strains of immune competent mouse and showed that xeno-rejection of hESC is T cell-mediated process (33). To test whether human immune system would reject undifferentiated hESC in vivo, authors transplanted human adult skin grafts, hESC and hESC-derived teratoma tissues under the kidney capsule of trimera mouse reconstituted with human PBMC (34) and showed that transplanted hESC and hESC teratoma tissues could form teratomas while transplanted tumor cells were rejected, concluding that the hESC did not trigger allogenic response from human immune system due to immune privileged properties (33). However, when pulsed with antigenic peptides, hESC were killed by antigen-specific $\mathrm{CD}^{+}$cytolytic T lymphocytes (CTL) (33).

Similar to the mESC, hESC lines have also been shown to exhibit immune privileged properties as they do not induce $\mathrm{T}$ cell proliferation in allogenic MLR (13), suppress differentiation and function of human DCs (35). Li et al. examined immune response against transplanted hESC in immune-compromised Prk $^{-/-}$mice and immune-competent CD1 mice and did not find any sign of immune-reactivity at the transplantation site, evident by lack of granulocytes infiltration in $\mathrm{Prk}^{-/-}$mice and abrogation of granulocyte and lymphocyte infiltration in immune competent mice upon endotoxin administration along with hESC, suggesting that the hESCs exhibit immunosuppressive properties in vivo (13). The hESCs failed to trigger T cell response in allogenic MLR assay and treatment with IFN- $\gamma$ to induce MHC I expression did 
not facilitate $\mathrm{T}$ cell activation by these cells, even upon fixation, suggesting that the hESC possess inherent immune-privileged properties (13). The immunosuppressive effect of hESC has been shown not to be contact dependent as hESC extracts could suppress differentiation and function of human DCs and it was not mediated by IL-10 or TGB- $\beta$ production (35). Production of arginase-I in tumor microenvironment in known to inhibit $\mathrm{T}$ cells by depleting L-arginine from the microenvironment (36) and the hESC-mediated immune suppression has also been shown to utilize this mechanism, as provision of L-arginine mitigates hESC-mediated $\mathrm{T}$ cell suppression (37). Utilizing humanized mice, Zhao et al. found that human fetal liver-derived iPSC lines engrafted in animals received some infiltration of reconstituted human immune cells; however, immune response against autologous hiPSC teratomas was much weaker than the allogenic hESC-derived teratomas (10). In addition, expression of CTLA-4-immunoglobulin (CTLA-4-Ig) and PD-L1 in hESC has also been recently shown to prevent their rejection in humanized mice, highlighting the involvement of immune mechanisms in rejection of hESC-induced teratomas (38).

As mentioned before, mouse iPSC lines derived from different somatic cell sources have been found to harbor somatic cell memory and exhibit differential differentiation profiles $(17,18)$ and despite the usefulness of animal models, significant differences exist between human and mouse physiology (22). Therefore, detailed characterization of the biology and the differentiation potential of human iPSC lines derived from different somatic cell sources is essential to identify the best somatic cell source and the best iPSC derivation method for generating human iPSC lines that exhibit little or no inherent immunogenicity. In this context, iPSC lines derived from human DCs represent an efficient model to characterize the inherent immunogenicity profile of human iPSC lines and their cellular derivatives (14), as DCs harbor well-characterized innate and adaptive immune mechanisms and they serve as the bridge between the innate and adaptive arms of the immune system (39-41). We have recently shown that human DC-derived iPSC lines do not express functional TLR, co-stimulatory molecules, or the antigen presentation machinery, and they fail to trigger TLR-mediated inflammatory cytokine response, inflammasome activation, and $\mathrm{T}$ cell activation in MLR assay (14). While DC-derived iPSC lines do express mRNAs of the innate and adaptive response intermediaries, these mRNAs are not translated into functional proteins, highlighting the critical role of DC lineage-specific transcription factors in this process (14). Furthermore, these iPSC lines do not express MHC class II molecules but do express low levels of MHC class I molecules (14), in agreement with findings in hESC lines $(13,33,42)$. Utilizing an iPSC line derived from human fibroblast, Lu et al. have also shown that it does not express MHC class II molecules or the co-stimulatory molecules and does not induce T cell proliferation in allogenic MLR (43). Interestingly, despite expressing minimal levels of MHC class I molecules, human DC-derived iPSC lines can efficiently present antigenic peptides to $\mathrm{T}$ cells, in agreement with findings in hESC (33). Figure 1 schematically shows the effect of reprogramming on innate and adaptive immune pathways of human peripheral blood-derived DCs (14).

\section{IMMUNOGENICITY OF PSC-DERIVED CELLULAR DERIVATIVES}

In their study showing minimal inherent immunogenicity of syngenic mouse iPSC lines, Araki et al. engrafted skin tissues as well as bone marrow from syngenic iPSC-derived animals and showed that the grafted cells and tissues were accepted by the host, and the bone marrow graft-derived T cells and B cells were also able to coexist with the host immune cells for over 5 months without any sign of immune cross-reactivity (7); however, authors did find immune infiltration at the site of cardiomyocyte injections (7), emphasizing the need to characterize the inherent immunogenicity of different somatic cell lineages and tissues derived from the iPSC lines. Guha et al. engrafted syngenic mouse iPSC, iPSC-derived EB, and cellular derivatives representing three germ layers, the neuronal cells (ectoderm), hepatocytes (endoderm), and endothelial cells (mesoderm), in the subcapsular renal space and did not find any sign of $\mathrm{T}$ cell reactivity against the grafts by immunohistochemistry and MLR (8). Morizane et al. transplantated autologous and allogenic iPSC-derived neural cells in non-human primates and found accumulation of $\mathrm{CD}_{4} 5^{+}$cells at the graft sites 3 months post injection in allograft recipient but not in the autologous graft recipients; however, both the autologous and allogenic grafted neurons survived in the recipients and no detectable IFN- $\gamma$ or TNF- $\alpha$ were found in the cerebrospinal fluid (CSF) ruling out an active immune response against the grafts (44). Utilizing mouse iPSC-derived endothelial cells (iEC) and comparable somatic cells, the aorta derived endothelial cells (AEC), de Almeida showed that undifferentiated iPSCs were rejected but the differentiated iEC survived and based on the similarities in single-cell transcriptomy profiles of the iEC and AEC, authors concluded that the iPSC-derived cellular derivatives develop immune tolerance properties comparable to self-tolerance mechanisms (11).

With regard to human PSC lines, hPSC-derived EB have been shown to inhibit $\mathrm{T}$ cell proliferation, similar to the hESC line (13). Utilizing EB derived from human DC-derived iPSC lines, we have also found that these EB do not express functional proteins for the TLRs, co-stimulatory molecules, or the antigen presentation pathway intermediaries (manuscript under preparation), in agreement with findings in hESC-derived EB (33). Utilizing humanized mice, Zhao et al. found that the smooth muscle cells are inherently more immunogenic than the retinal pigment epithelium, and it correlates with their immune-antigen profiles (10). Taken together, while most of these studies have reported minimal or no immunogenicity of autologous iPSCderived cellular derivatives in transplanted animals, differences in the immunogenicity profiles of different somatic cells highlight the need to develop efficient models to systematically address this issue. Toward this, detailed characterization of human DC-derived iPSC lines, EB generated from these iPSC lines and more importantly functional APC derived from them could provide useful insights not only toward the inherent immunogenicity profile of human iPSC lines and their cellular derivatives, but also toward development of innate and adaptive immune mechanisms in human DC lineage. 


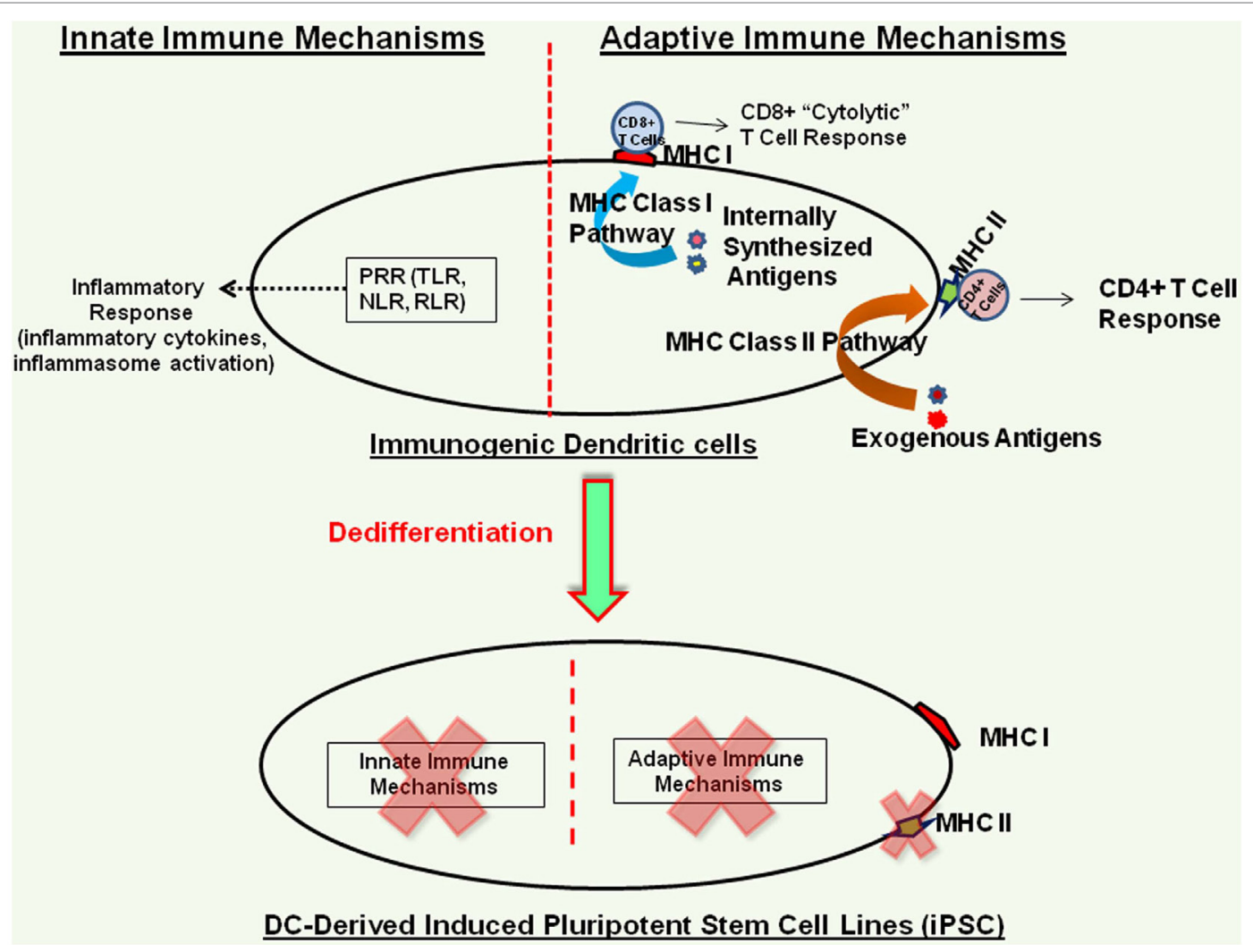

FIGURE 1 | Immunogenicity profile of human terminally differentiated dendritic cell (DC)-derived induced pluripotent stem cell (iPSC) lines. Dedifferentiation of human DCs effectively shuts down their innate and adaptive immune mechanisms; however, these iPSC lines do express low levels of MHC class I molecules (14).

\section{POTENTIAL MECHANISMS FOR REJECTION OF PSC-INDUCED TERATOMA}

Among the key immune effectors facilitating the development of protective host immune response includes DCs that orchestrate innate and adaptive immune responses and also control selfreactive immune responses, $\mathrm{T}$ cells that provide long-lasting cellular immunity, B cells that modulate humoral immunity, and NK cells that target cells lacking MHC molecules. As discussed before, hESC negatively modulate human DC differentiation and function in a contact-independent manner (35). With regard to interaction with $\mathrm{T}$ cells, hESC and human iPSC lines do not trigger $\mathrm{T}$ cell activation in MLR and suppress $\mathrm{T}$ cell function by downregulating the production of effector cytokines and cytolytic activity $(13,14)$. However, as mentioned before, despite expressing minimal amount of MHC class I molecules $(13,14$, 42), hESC and human iPSC lines can efficiently present antigenic peptides to $\mathrm{T}$ cells $(14,33,43)$. While IL-2-activated mouse NK cells have been shown to kill mESC (16), human hESC are not killed by the NK cells as they do not express NK cell ligands (42). Figure 2 summarizes interaction of hPSC lines with different immune effectors.

Of note, in normal physiology, PSC do not directly interact with immune effectors and the host immunosuppressive mechanisms prevent immune rejection of developing embryo that harbors $50 \%$ foreign DNA. It is possible that the presence of immune infiltrating cells in PSC induced teratomas is due to natural immune surveillance phenomenon and these immune effectors would "ignore" the transplanted PSC and their cellular derivatives due to lack of "non-self antigens" or the "danger signals" or due to immunosuppressive properties of the PSC, as grafted bone marrow-derived T cells and B cells coexist with host immune cells without any sign of immune reactivity (7) and no inflammatory cytokines were detectable in the CSF of neuronal transplants (44). While these findings are encouraging, we now discuss some of the potential mechanisms that might be contributing to the rejection of transplanted ESCs and iPSCs in mouse models (Figure 3). Among these include presentation of antigenic peptides acquired by the transplanted hPSC from their microenvironment to cytolytic T cells that can kill these hPSC in antigen-specific manner $(14,43)$. Mouse PSC have also been shown to be susceptible to alternative complement pathwaymediated killing that affects their ability to form teratomas, in an inverse correlation to the number of PSC transplanted as complement could block teratoma formation by $1 \times 10^{5}$ but not with $1 \times 10^{6} \mathrm{PSC}$, and the $\mathrm{C} 3^{-/-}$mice could form teratomas much faster than the $\mathrm{C}^{+/+}$mice in part due to deficiency of sialic acid in the PSC (45). In addition, while hESC and iPSC lines do not express MHC II molecules, it is possible that the MHC class II 


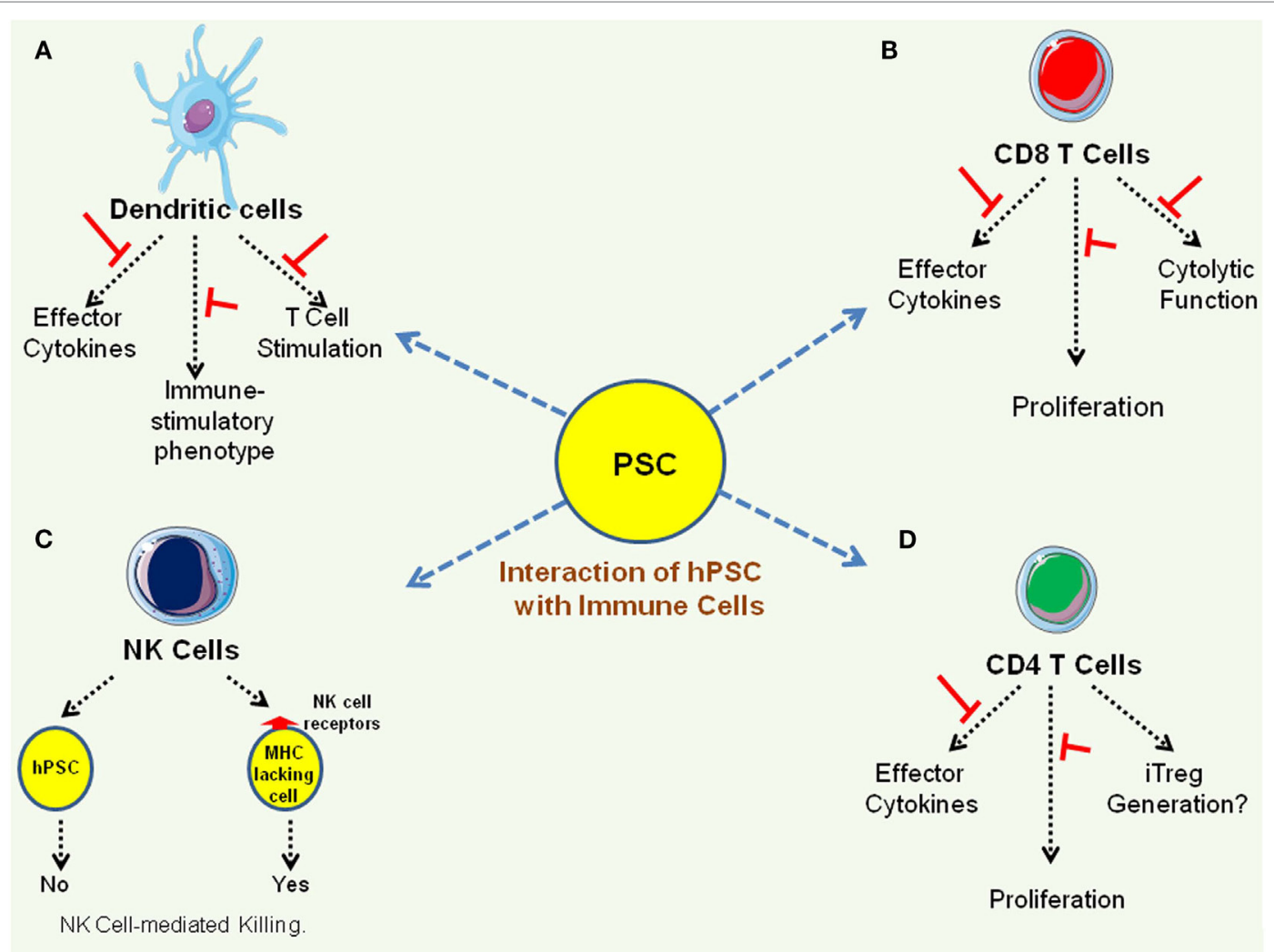

FIGURE 2 | Interaction of immune effectors with human pluripotent stem cell (hPSC) lines. (A) Pluripotent stem cell (PSC) suppresses immune stimulatory properties of dendritic cells $(35,37)$. (B,C) PSC suppresses effector function of CD8 T cells as well as CD4 T cells (13, 14). (D). While PSC express very low levels of MHC class I molecules and mouse PSC have been shown to be susceptible to natural killer (NK) cell-mediated killing (16), human PSC are not killed by NK cells as they do not express NK cell receptors (42).

molecules in hPSC are improperly processed and are eventually degraded, thereby generating a repertoire of peptide epitopes that along with other hPSC-derived proteins are acquired by the host APC from apoptotic hPSC through well-known mechanisms (46) and are presented to T cells facilitating an allogenic response, a phenomenon known as indirect allogenic recognition (47).

\section{STRATEGIES TO OVERCOME INHERENT IMMUNOGENICITY OF PSC}

Immunosuppressive drugs (48) as well as immune modulators inhibiting $\mathrm{T}$ cell activation (49) have been shown to facilitate acceptance of hESC xeno-transplants in mouse models. Swijnenburg et al. utilized clinically available immunosuppressive drugs, calcinurin inhibitor [tacrolimus (TAC)], target of rapamycin inhibitor [sirolimus (SIR)], and antiproliferative agent (mycophenolate mofetil) and showed that the combination of SIR and TAC could facilitate long-term survival of transplanted hESC by immunosuppressive effect on both the cellular as well as humoral arms of the host immune system (48). However, despite improving the survival of transplanted hESC, it could not facilitate their engraftment beyond 28 days (48). Pearl et al. showed that the combination of CTLA-4-Ig fusion protein, anti-CD40 ligand (anti-CD40L) and anti-lymphocyte functionassociated antigen-1 (anti-LFA-1) antibody-mediated shortterm immune suppression, to block $\mathrm{T}$ cell activation molecules and to provide inhibitory signals, could facilitate engraftment of allogenic/xenogenic hESC as well as iPSC beyond 28 days, through immunosuppressive effect on $\mathrm{CD}^{+}$and $\mathrm{CD} 8^{+}$effector $\mathrm{T}$ cells and induction of regulatory $\mathrm{T}$ cells (49). As mentioned before, expression of CTL-A4-Ig and PD-L1 in hESC has also been shown to protect them from immune rejection in humanized mice (38); however, it should be emphasized here that constitutive expression of these molecules might not be a good strategy for engrafting iPSC-derived immune cells, as it could compromise their effector function profiles. Tolerance to allografts has also been achieved in animal models through mixed hematopoietic chimerism, whereby donor HSC are engrafted in the host under immunosuppressive conditions and the cells derived from these HSC orchestrate tolerance to allografts (50). In addition, incorporation of regulatory $\mathrm{T}$ cells is also known to facilitate allograft acceptance (51).

In summary, while donor-specific human iPSC lines have been shown to exhibit immune suppressive properties and 

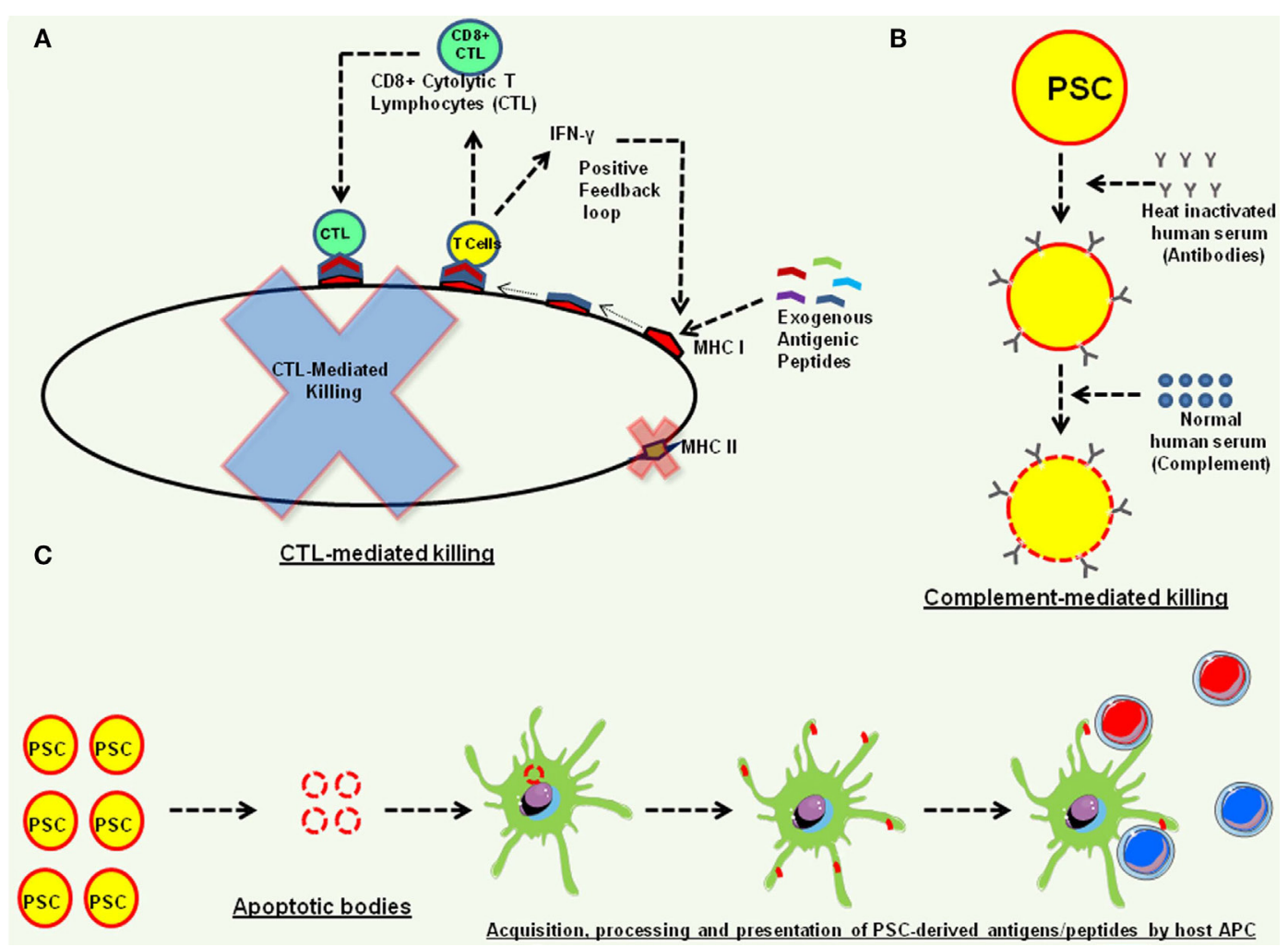

FIGURE 3 | Potential mechanisms of teratoma rejection in animal models. (A) The human embryonic stem cell and human induced pluripotent stem cell (iPSC) express low levels of MHC class I molecules $(13,33,42)$ that can efficiently acquire antigenic peptides from their microenvironment and present them to CD ${ }^{+}$ cytolytic T lymphocytes (CTL) that produce effector cytokines, such as interferon- $\gamma$ (IFN- $\gamma$ ), and also exhibit cytolytic function and kill these pluripotent stem cell (PSC) $(14,43)$. The IFN- $\gamma$ can also further induce the expression of MHC class I molecules on human PSC, forming a positive feedback loop (14). (B) Complementmediated killing of PSC. Human PSC are susceptible to alternative complement pathway-mediated killing (45). (C) Indirect allo-recognition. Transplanted PSC undergoing apoptosis can be phagocytosed by host antigen-presenting cells, and PSC acquired proteins can be processed and presented to host T cells for alloresponse.

several ESC and iPSC-derived cellular derivatives have been successfully transplanted in animal models without immunological complications, it is possible to overcome host immune rejection mechanisms to facilitate engraftment of iPSC-derived cells/ tissues (Figure 4). However, it would be best to optimize the iPSC-derivation methodologies and identify best somatic cells to generate donor-specific iPSC lines that exhibit little or no inherent immunogenicity. Detailed characterization of the inherent immunogenicity profiles of iPSC-derived somatic cell lineages is also essential to incorporate best immunosuppressive strategy to facilitate their engraftment.

\section{IMMUNOGENIC FUNCTIONALITY OF PSC-DERIVED IMMUNE CELLS}

Although donor-specific iPSC lines and their cellular derivatives are not expected to be inherently immunogenic, iPSC-derived cellular derivatives are expected to be fully functional, which in the case of iPSC-derived immune cells is orchestration of all the immunological parameters associated with the immune cell lineage of choice. Donor-derived immune cells, such as DC and
T cells have been used to develop DC-based cancer vaccines and tumor antigen-specific $\mathrm{T}$ cell receptor (TCR)/chimeric antigen receptor (CAR) engineered antitumor $\mathrm{T}$ cells that have produced remarkable clinical outcomes in cancer patients (52-58). However, since donor-derived mature T cells possess endogenous TCR and the DC derived from cancer patients might be compromised in their immunogenicity profile due to tumor-induced immune suppression mechanisms, availability of donor-specific naive immune effectors with customized functional profile could significantly improve the efficacy of current immunotherapy approaches $(58,59)$. In this context, iPSC-derived immunogenic APC would be very useful for developing personalized cancer immunotherapy approaches, not only as standalone cancer vaccines but also as an adjuvant for $\mathrm{T}$ cell-based adaptive immunotherapy approaches. Donorspecific tolerogenic APC on the other hand would be useful for the treatment of autoimmune diseases. Similarly, donor-specific naive antitumor $\mathrm{T}$ cells, generated with TCR- or CAR-based approaches, could improve the efficacy of adoptive transfer-based cancer immunotherapy approaches $(58,60,61)$. This would be of special significance for the elderly where the quantity as well as the quality of immune effectors is compromised with age. 

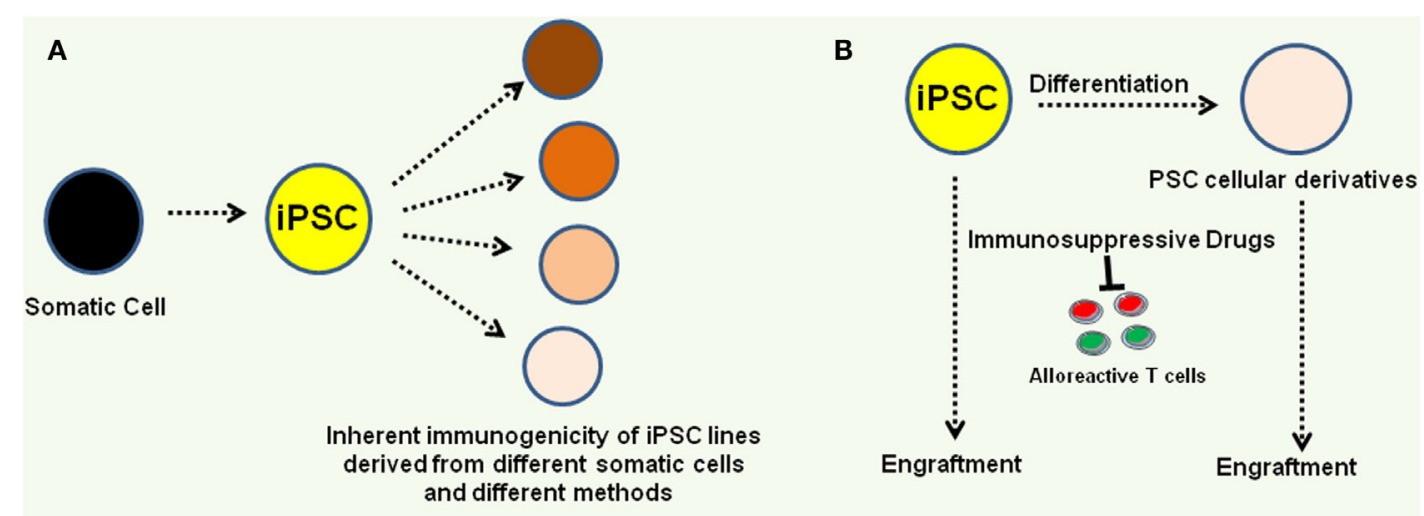

\section{C}
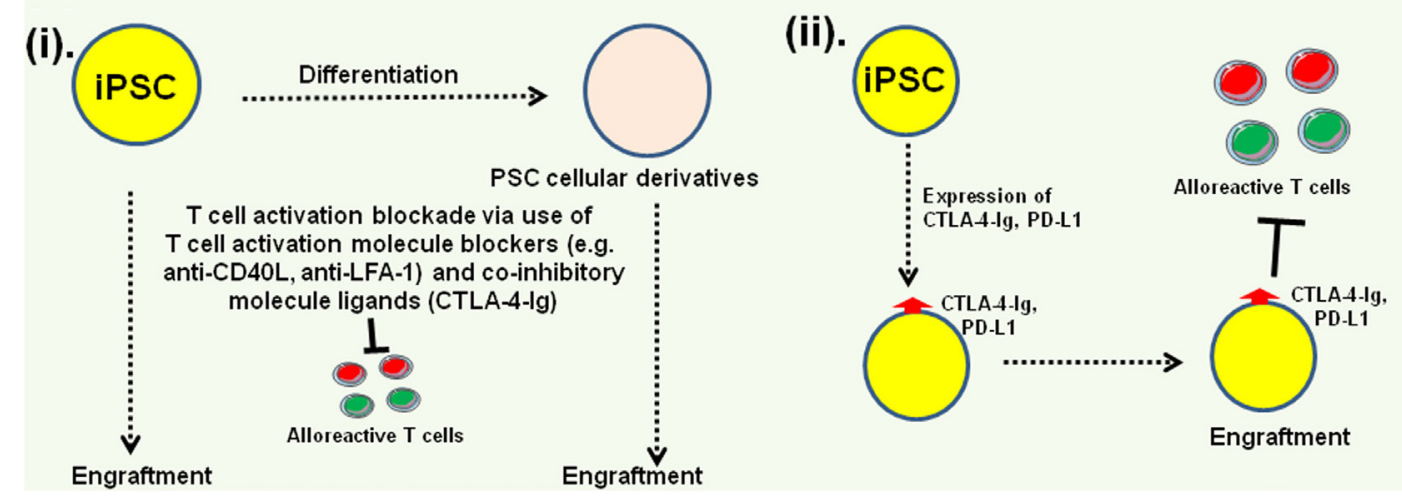

FIGURE 4 | Approaches to overcome potential immunogenicity of induced pluripotent stem cell (iPSC) and their derivatives. (A) Identification of best iPSC derivation methods and best somatic cells for generating iPSC lines that exhibit no or very low inherent immunogenicity profiles. (B) Incorporation of immunosuppressive drugs can facilitate pluripotent stem cell (PSC) engraftment (48). (C) Blocking immune cell activation can also facilitate PSC rejection (49). Among the approaches, T cell activating molecule blockers [e.g., anti-CD40 ligand (anti-CD4OL), anti-lymphocyte function-associated antigen-1 (anti-LFA-1)], and co-inhibitory molecule activators [CTLA-4-immunoglobulin (CTLA-4-lg)] could be administered as adjuvants (i) or co-inhibitory molecule ligands (e.g., CTLA-4-Ig, PD-L1) could be expressed on the PSC (ii) to engage co-inhibitory molecules on host T cells (38).

A significant progress has been made toward generating effector T cells, APC, NK cells, and other blood lineages from the hESC and iPSC lines (62-65). Functional APC-derived from ESC and iPSC lines have been shown to effectively acquire and process the antigens and present antigenic peptide epitopes to T cells through traditional antigen presentation pathways as well as through cross-presentation routes $(62,66,67)$. Customized antitumor T cells have also been derived from hESC and iPSC lines via tumor antigen-specific TCR and CAR engineering approaches, and these $\mathrm{T}$ cells have been shown to exhibit antigen-specific effector function (68-70). Donor-specific NK cells have also been derived from hESC and iPSC lines and these cells have also been shown to exhibit effector function in vitro as well as in vivo $(63,71)$. While fibroblasts remain one of the preferred cells for derivation of donor-specific iPSC lines, as mentioned before, lineage specific models could be useful to systematically characterize the inherent immune-reactivity of hPSC lines and somatic cells derived from them, and also for characterizing the molecular and functional profile of iPSC-derived somatic cells. For example, as mentioned before, human DC-derived iPSC lines have provided useful insights toward the inherent immunogenicity profile of human
iPSC lines (14) and the iPSC-APC derived from them could also be useful for systematically characterizing their molecular as well as functional profile, utilizing autologous human DC as controls.

\section{CONCLUSION}

Since the isolation of first hESC lines and especially the derivation of donor-specific iPSC lines, regenerative medicine field has progressed at an exponential pace. Methods have been developed to generate donor-specific iPSC lines that are free of integrating viral vectors from different somatic cell lineages, including terminally differentiated primary immune cells, and different types of functional somatic cells have been derived, some of which have even advanced to the clinical trials stage. However, several challenges, such as the inherent immunogenicity of iPSC lines, have also been identified that need to be addressed. Since iPSC lines derived from different somatic cells and with different iPSC derivation methods can harbor different genetic and epigenetic signatures, it is necessary to fully characterize the mechanism of development and maintenance of pluripotency to generate iPSC lines with stable genomic architecture. This is important given 
that incomplete reprogramming could generate a neoantigen repertoire that could translate into enhanced immunogenicity of iPSC lines. Furthermore, while inherent immunogenicity of iPSC lines is not desired, iPSC-derived immune cells need to be fully functional. Therefore, according to the somatic cell lineage of choice, appropriate models need to be developed to characterize their molecular, cellular, as well as functional profiles. Addressing these issues is critical for developing safe and effective CRT.

\section{AUTHOR CONTRIBUTIONS}

AC conceived and wrote the manuscript.

\section{REFERENCES}

1. Thomson JA, Itskovitz-Eldor J, Shapiro SS, Waknitz MA, Swiergiel JJ, Marshall VS, et al. Embryonic stem cell lines derived from human blastocysts. Science (1998) 282:1145-7. doi:10.1126/science.282.5391.1145

2. Takahashi K, Yamanaka S. Induction of pluripotent stem cells from mouse embryonic and adult fibroblast cultures by defined factors. Cell (2006) 126:663-76. doi:10.1016/j.cell.2006.07.024

3. Takahashi K, Tanabe K, Ohnuki M, Narita M, Ichisaka T, Tomoda K, et al. Induction of pluripotent stem cells from adult human fibroblasts by defined factors. Cell (2007) 131:861-72. doi:10.1016/j.cell.2007.11.019

4. Yu J, Vodyanik MA, Smuga-Otto K, Antosiewicz-Bourget J, Frane JL, Tian S, et al. Induced pluripotent stem cell lines derived from human somatic cells. Science (2007) 318:1917-20. doi:10.1126/science.1151526

5. Zhao T, Zhang ZN, Rong Z, Xu Y. Immunogenicity of induced pluripotent stem cells. Nature (2011) 474:212-5. doi:10.1038/nature10135

6. Okita K, Nagata N, Yamanaka S. Immunogenicity of induced pluripotent stem cells. Circ Res (2011) 109:720-1. doi:10.1161/RES.0b013e318232e187

7. Araki R, Uda M, Hoki Y, Sunayama M, Nakamura M, Ando S, et al. Negligible immunogenicity of terminally differentiated cells derived from induced pluripotent or embryonic stem cells. Nature (2013) 494:100-4. doi:10.1038/ nature11807

8. Guha P, Morgan JW, Mostoslavsky G, Rodrigues NP, Boyd AS. Lack of immune response to differentiated cells derived from syngeneic induced pluripotent stem cells. Cell Stem Cell (2013) 12:407-12. doi:10.1016/j. stem.2013.01.006

9. Todorova D, Kim J, Hamzeinejad S, He J, Xu Y. Brief report: immune microenvironment determines the immunogenicity of induced pluripotent stem cell derivatives. Stem Cells (2016) 34:510-5. doi:10.1002/stem.2227

10. Zhao T, Zhang ZN, Westenskow PD, Todorova D, Hu Z, Lin T, et al. Humanized mice reveal differential immunogenicity of cells derived from autologous induced pluripotent stem cells. Cell Stem Cell (2015) 17:353-9. doi:10.1016/j. stem.2015.07.021

11. de Almeida PE, Meyer EH, Kooreman NG, Diecke S, Dey D, SanchezFreire V, et al. Transplanted terminally differentiated induced pluripotent stem cells are accepted by immune mechanisms similar to self-tolerance. Nat Commun (2014) 5:3903. doi:10.1038/ncomms4903

12. Koch CA, Geraldes P, Platt JL. Immunosuppression by embryonic stem cells. Stem Cells (2008) 26:89-98. doi:10.1634/stemcells.2007-0151

13. Li L, Baroja ML, Majumdar A, Chadwick K, Rouleau A, Gallacher L, et al. Human embryonic stem cells possess immune-privileged properties. Stem Cells (2004) 22:448-56. doi:10.1634/stemcells.22-4-448

14. Chhabra A, Chen IP, Batra D. Human dendritic cell-derived induced pluripotent stem cell lines are not immunogenic. J Immunol (2017) 198(5):1875-86. doi:10.4049/jimmunol.1601676

15. Magliocca JF, Held IK, Odorico JS. Undifferentiated murine embryonic stem cells cannot induce portal tolerance but may possess immune privilege secondary to reduced major histocompatibility complex antigen expression. Stem Cells Dev (2006) 15:707-17. doi:10.1089/scd.2006.15.707

16. Dressel R, Nolte J, Elsner L, Novota P, Guan K, Streckfuss-Bomeke K, et al. Pluripotent stem cells are highly susceptible targets for syngeneic, allogeneic, and xenogeneic natural killer cells. FASEB J (2010) 24:2164-77. doi:10.1096/ f. $09-134957$

\section{ACKNOWLEDGMENTS}

Author thanks all the authors of the manuscripts that are cited here and many more that could not be included due to space limitations. AC conceived and wrote the manuscript. AC conceived and made the figures, in part using the Servier-Medical Art Slides. The author also acknowledges help from Deepika Batra and Feny Rasania in preparation of the manuscript. The author has no competing interest associated with this manuscript. Grant support from the State of Connecticut Regenerative Medicine Program (10-SCA-23 and 13-SCB-05) is also duly acknowledged.

17. Kim K, Doi A, Wen B, Ng K, Zhao R, Cahan P, et al. Epigenetic memory in induced pluripotent stem cells. Nature (2010) 467:285-90. doi:10.1038/ nature 09342

18. Vaskova EA, Stekleneva AE, Medvedev SP, Zakian SM. "Epigenetic memory" phenomenon in induced pluripotent stem cells. Acta Naturae (2013) 5:15-21.

19. Schnerch A, Cerdan C, Bhatia M. Distinguishing between mouse and human pluripotent stem cell regulation: the best laid plans of mice and men. Stem Cells (2010) 28:419-30. doi:10.1002/stem.298

20. Ginis I, Luo Y, Miura T, Thies S, Brandenberger R, Gerecht-Nir S, et al. Differences between human and mouse embryonic stem cells. Dev Biol (2004) 269:360-80. doi:10.1016/j.ydbio.2003.12.034

21. Suh MR, Lee Y, Kim JY, Kim SK, Moon SH, Lee JY, et al. Human embryonic stem cells express a unique set of microRNAs. Dev Biol (2004) 270:488-98. doi:10.1016/j.ydbio.2004.02.019

22. Mestas J, Hughes CC. Of mice and not men: differences between mouse and human immunology. J Immunol (2004) 172:2731-8. doi:10.4049/ jimmunol.172.5.2731

23. Sitnicka E, Buza-Vidas N, Larsson S, Nygren JM, Liuba K, Jacobsen SE. Human CD34+ hematopoietic stem cells capable of multilineage engrafting NOD/SCID mice express flt3: distinct flt 3 and c-kit expression and response patterns on mouse and candidate human hematopoietic stem cells. Blood (2003) 102:881-6. doi:10.1182/blood-2002-06-1694

24. Tokugawa Y, Koyama M, Silver J. A molecular basis for species differences in Thy-1 expression patterns. Mol Immunol (1997) 34:1263-72. doi:10.1016/ S0161-5890(98)00010-8

25. Peschon JJ, Morrissey PJ, Grabstein KH, Ramsdell FJ, Maraskovsky E, Gliniak BC, et al. Early lymphocyte expansion is severely impaired in interleukin 7 receptor-deficient mice. J Exp Med (1994) 180:1955-60. doi:10.1084/ jem.180.5.1955

26. Roifman CM, Zhang J, Chitayat D, Sharfe N. A partial deficiency of interleukin-7R alpha is sufficient to abrogate T-cell development and cause severe combined immunodeficiency. Blood (2000) 96:2803-7.

27. Elder ME, Skoda-Smith S, Kadlecek TA, Wang F, Wu J, Weiss A. Distinct $T$ cell developmental consequences in humans and mice expressing identical mutations in the DLAARN motif of ZAP-70. J Immunol (2001) 166:656-61. doi:10.4049/jimmunol.166.1.656

28. Tibbetts MD, Zheng L, Lenardo MJ. The death effector domain protein family: regulators of cellular homeostasis. Nat Immunol (2003) 4:404-9. doi:10.1038/ ni0503-404

29. Chun HJ, Zheng L, Ahmad M, Wang J, Speirs CK, Siegel RM, et al. Pleiotropic defects in lymphocyte activation caused by caspase- 8 mutations lead to human immunodeficiency. Nature (2002) 419:395-9. doi:10.1038/nature01063

30. Lublin FD, Knobler RL, Kalman B, Goldhaber M, Marini J, Perrault M, et al. Monoclonal anti-gamma interferon antibodies enhance experimental allergic encephalomyelitis. Autoimmunity (1993) 16:267-74. doi:10.3109/ 08916939309014645

31. Panitch HS, Hirsch RL, Haley AS, Johnson KP. Exacerbations of multiple sclerosis in patients treated with gamma interferon. Lancet (1987) 1:893-5 doi:10.1016/S0140-6736(87)92863-7

32. Drukker M. Immunogenicity of human embryonic stem cells: can we achieve tolerance? Springer Semin Immunopathol (2004) 26:201-13. doi:10.1007/ s00281-004-0163-5 
33. Drukker M, Katchman H, Katz G, Even-Tov Friedman S, Shezen E, Hornstein E, et al. Human embryonic stem cells and their differentiated derivatives are less susceptible to immune rejection than adult cells. Stem Cells (2006) 24:221-9. doi:10.1634/stemcells.2005-0188

34. Segall H, Lubin I, Marcus H, Canaan A, Reisner Y. Generation of primary antigen-specific human cytotoxic T lymphocytes in human/mouse radiation chimera. Blood (1996) 88:721-30.

35. Mohib K, Allan D, Wang L. Human embryonic stem cell-extracts inhibit the differentiation and function of monocyte-derived dendritic cells. Stem Cell Rev (2010) 6:611-21. doi:10.1007/s12015-010-9185-7

36. Rodriguez PC, Quiceno DG, Zabaleta J, Ortiz B, Zea AH, Piazuelo MB, et al. Arginase I production in the tumor microenvironment by mature myeloid cells inhibits T-cell receptor expression and antigen-specific T-cell responses. Cancer Res (2004) 64:5839-49. doi:10.1158/0008-5472.CAN-04-0465

37. Yachimovich-Cohen N, Even-Ram S, Shufaro Y, Rachmilewitz J, Reubinoff B. Human embryonic stem cells suppress $\mathrm{T}$ cell responses via arginase I-dependent mechanism. J Immunol (2010) 184:1300-8. doi:10.4049/ jimmunol.0804261

38. Rong Z, Wang M, Hu Z, Stradner M, Zhu S, Kong H, et al. An effective approach to prevent immune rejection of human ESC-derived allografts. Cell Stem Cell (2014) 14:121-30. doi:10.1016/j.stem.2013.11.014

39. Schuurhuis DH, Fu N, Ossendorp F, Melief CJ. Ins and outs of dendritic cells. Int Arch Allergy Immunol (2006) 140:53-72. doi:10.1159/000092002

40. Merad M, Sathe P, Helft J, Miller J, Mortha A. The dendritic cell lineage: ontogeny and function of dendritic cells and their subsets in the steady state and the inflamed setting. Annu Rev Immunol (2013) 31:563-604. doi:10.1146/ annurev-immunol-020711-074950

41. Steinman RM. Decisions about dendritic cells: past, present, and future. Annu Rev Immunol (2012) 30:1-22. doi:10.1146/annurev-immunol-100311-102839

42. Drukker M, Katz G, Urbach A, Schuldiner M, Markel G, Itskovitz-Eldor J, et al. Characterization of the expression of MHC proteins in human embryonic stem cells. Proc Natl Acad Sci U S A (2002) 99:9864-9. doi:10.1073/ pnas.142298299

43. Lu Q, Yu M, Shen C, Chen X, Feng T, Yao Y, et al. Negligible immunogenicity of induced pluripotent stem cells derived from human skin fibroblasts. PLoS One (2014) 9:e114949. doi:10.1371/journal.pone.0114949

44. Morizane A, Doi D, Kikuchi T, Okita K, Hotta A, Kawasaki T, et al. Direct comparison of autologous and allogeneic transplantation of iPSC-derived neural cells in the brain of a non-human primate. Stem Cell Reports (2013) 1:283-92. doi:10.1016/j.stemcr.2013.08.007

45. Koch CA, Jordan CE, Platt JL. Complement-dependent control of teratoma formation by embryonic stem cells. J Immunol (2006) 177:4803-9. doi:10.4049/ jimmunol.177.7.4803

46. Albert ML, Sauter B, Bhardwaj N. Dendritic cells acquire antigen from apoptotic cells and induce class I-restricted CTLs. Nature (1998) 392:86-9. doi:10.1038/32183

47. Kreisel D, Krupnick AS, Gelman AE, Engels FH, Popma SH, Krasinskas AM, et al. Non-hematopoietic allograft cells directly activate CD8+ T cells and trigger acute rejection: an alternative mechanism of allorecognition. Nat Med (2002) 8:233-9. doi:10.1038/nm0302-233

48. Swijnenburg RJ, Schrepfer S, Govaert JA, Cao F, Ransohoff K, Sheikh AY, et al. Immunosuppressive therapy mitigates immunological rejection of human embryonic stem cell xenografts. Proc Natl Acad Sci U S A (2008) 105:12991-6. doi:10.1073/pnas.0805802105

49. Pearl JI, Lee AS, Leveson-Gower DB, Sun N, Ghosh Z, Lan F, et al. Shortterm immunosuppression promotes engraftment of embryonic and induced pluripotent stem cells. Cell Stem Cell (2011) 8:309-17. doi:10.1016/j. stem.2011.01.012

50. Gibbons C, Sykes M. Manipulating the immune system for anti-tumor responses and transplant tolerance via mixed hematopoietic chimerism. Immunol Rev (2008) 223:334-60. doi:10.1111/j.1600-065X.2008.00636.x

51. Waldmann H, Chen TC, Graca L, Adams E, Daley S, Cobbold S, et al. Regulatory $\mathrm{T}$ cells in transplantation. Semin Immunol (2006) 18:111-9. doi:10.1016/j.smim.2006.01.010

52. Chhabra A, Yang L, Wang P, Comin-Anduix B, Das R, Chakraborty NG, et al. CD4+CD25- $\mathrm{T}$ cells transduced to express MHC class I-restricted epitope-specific TCR synthesize Th1 cytokines and exhibit MHC class I-restricted cytolytic effector function in a human melanoma model. J Immunol (2008) 181:1063-70. doi:10.4049/jimmunol.181.2.1063
53. Mukherji B, Chakraborty NG, Yamasaki S, Okino T, Yamase H, Sporn JR, et al. Induction of antigen-specific cytolytic $\mathrm{T}$ cells in situ in human melanoma by immunization with synthetic peptide-pulsed autologous antigen presenting cells. Proc Natl Acad Sci U S A (1995) 92:8078-82. doi:10.1073/pnas.92. 17.8078

54. Dudley ME, Wunderlich JR, Robbins PF, Yang JC, Hwu P, Schwartzentruber DJ, et al. Cancer regression and autoimmunity in patients after clonal repopulation with antitumor lymphocytes. Science (2002) 298:850-4. doi:10.1126/science.1076514

55. Morgan RA, Dudley ME, Wunderlich JR, Hughes MS, Yang JC, Sherry RM, et al. Cancer regression in patients after transfer of genetically engineered lymphocytes. Science (2006) 314(5796):126-9. doi:10.1126/science.1129003

56. Gilboa E. DC-based cancer vaccines. J Clin Invest (2007) 117:1195-203. doi:10.1172/JCI31205

57. Kalos M, Levine BL, Porter DL, Katz S, Grupp SA, Bagg A, et al. T cells with chimeric antigen receptors have potent antitumor effects and can establish memory in patients with advanced leukemia. Sci Transl Med (2011) 3:95ra73. doi:10.1126/scitranslmed.3002842

58. Chhabra A. TCR-engineered, customized, antitumor $\mathrm{T}$ cells for cancer immunotherapy: advantages and limitations. ScientificWorldJournal (2011) 11:121-9. doi:10.1100/tsw.2011.10

59. Rosenberg SA, Yang JC, Restifo NP. Cancer immunotherapy: moving beyond current vaccines. Nat Med (2004) 10:909-15. doi:10.1038/nm1100

60. Ray S, Chhabra A, Mehrotra S, Chakraborty NG, Ribas A, Economou J, et al. Obstacles to and opportunities for more effective peptide-based therapeutic immunization in human melanoma. Clin Dermatol (2009) 27:603-13. doi:10.1016/j.clindermatol.2008.09.019

61. Chhabra A, Chakraborty NG, Mukherji B. Silencing of endogenous IL-10 in human dendritic cells leads to the generation of an improved CTL response against human melanoma associated antigenic epitope, MART-1 27-35. Clin Immunol (2008) 126:251-9. doi:10.1016/j.clim.2007.11.011

62. Zhan X, Dravid G, Ye Z, Hammond H, Shamblott M, Gearhart J, et al. Functional antigen-presenting leucocytes derived from human embryonic stem cells in vitro. Lancet (2004) 364:163-71. doi:10.1016/S0140-6736(04) 16629-4

63. Woll PS, Grzywacz B, Tian X, Marcus RK, Knorr DA, Verneris MR, et al. Human embryonic stem cells differentiate into a homogeneous population of natural killer cells with potent in vivo antitumor activity. Blood (2009) 113:6094-101. doi:10.1182/blood-2008-06-165225

64. Slukvin II, Vodyanik MA, Thomson JA, Gumenyuk ME, Choi KD. Directed differentiation of human embryonic stem cells into functional dendritic cells through the myeloid pathway. J Immunol (2006) 176:2924-32. doi:10.4049/ jimmunol.176.5.2924

65. Galic Z, Kitchen SG, Kacena A, Subramanian A, Burke B, Cortado R, et al. T lineage differentiation from human embryonic stem cells. Proc Natl Acad Sci U S A (2006) 103:11742-7. doi:10.1073/pnas.0604244103

66. Tseng SY, Nishimoto KP, Silk KM, Majumdar AS, Dawes GN, Waldmann H, et al. Generation of immunogenic dendritic cells from human embryonic stem cells without serum and feeder cells. Regen Med (2009) 4:513-26. doi:10.2217/ rme.09.25

67. Silk KM, Silk JD, Ichiryu N, Davies TJ, Nolan KF, Leishman AJ, et al. Cross-presentation of tumour antigens by human induced pluripotent stem cell-derived CD141(+)XCR1+ dendritic cells. Gene Ther (2012) 19:1035-40. doi:10.1038/gt.2011.177

68. Themeli M, Kloss CC, Ciriello G, Fedorov VD, Perna F, Gonen M, et al. Generation of tumor-targeted human $\mathrm{T}$ lymphocytes from induced pluripotent stem cells for cancer therapy. Nat Biotechnol (2013) 31:928-33. doi:10.1038/nbt.2678

69. Nishimura T, Kaneko S, Kawana-Tachikawa A, Tajima Y, Goto H, Zhu D, et al. Generation of rejuvenated antigen-specific $\mathrm{T}$ cells by reprogramming to pluripotency and redifferentiation. Cell Stem Cell (2013) 12:114-26. doi:10.1016/j.stem.2012.11.002

70. Vizcardo R, Masuda K, Yamada D, Ikawa T, Shimizu K, Fujii S, et al. Regeneration of human tumor antigen-specific T cells from iPSCs derived from mature CD8(+) T cells. Cell Stem Cell (2013) 12:31-6. doi:10.1016/j. stem.2012.12.006

71. Knorr DA, Kaufman DS. Pluripotent stem cell-derived natural killer cells for cancer therapy. Transl Res (2010) 156:147-54. doi:10.1016/j.trsl.2010. 07.008 
Conflict of Interest Statement: The author declares that the research was conducted in the absence of any commercial or financial relationships that could be construed as a potential conflict of interest.

The reviewer, FR, and handling editor declared their shared affiliation, and the handling editor states that the process nevertheless met the standards of a fair and objective review.
Copyright (C) 2017 Chhabra. This is an open-access article distributed under the terms of the Creative Commons Attribution License (CC BY). The use, distribution or reproduction in other forums is permitted, provided the original author(s) or licensor are credited and that the original publication in this journal is cited, in accordance with accepted academic practice. No use, distribution or reproduction is permitted which does not comply with these terms. 